\title{
Los bosques de Abies guatemalensis Rehder de San Marcos, Guatemala: una oportunidad para su restauración ecológica
}

\author{
José V. Martínez-Arévalo* \\ Subárea de Ciencias Biológicas, Facultad de Agronomía, Universidad de San Carlos de Guatemala, Guatemala \\ *Autor al que se dirige la correspondencia: josevm2000@yahoo.com \\ Recibido: 17 de diciembre 2015 / Revisión: 30 de marzo 2016 / Aceptado: 30 de junio 2016 / Disponible en línea: 01 de agosto 2016
}

\section{Resumen}

— l pinabete, Abies guatemalensis Rehder, es una especie distribuida en las montañas altas de Guatemala. Según el 1 Consejo Nacional de Áreas Protegidas (Conap), sus poblaciones se encuentran severamente fragmentadas. Por su rareza y fragmentación, esta especie se encuentra protegida por el Convenio sobre el Comercio Internacional de Especies Amenazadas de Fauna y Flora Silvestre (Cites), lo que pone en relieve la importancia de la recuperación de sus bosques para su debida conservación. El éxito de su establecimiento puede mejorase al sembrarla junto a plantas nodriza, que le ayudarían a sobrevivir a las condiciones secas del año y las heladas que se presentan en las zonas de mayor altitud del país durante los meses de noviembre a febrero. Este trabajo muestra las características físicas, químicas y de vegetación del área de tres bosques de pinabete (A. guatemalensis) de la parte alta de San Marcos. Además, presenta la propuesta de restauración ecológica estimulada simulando la sucesión vegetal del área. Se elaboraron mapas, y describieron tres bosques de pinabete y sus alrededores; se presenta el estudio de la composición química del suelo y la vegetación y su representación en diferentes etapas de sucesión vegetal. Además, se propone la recuperación de áreas alrededor de parches de bosque, consistente en establecer plantas arbustivas en lugares abiertos donde aún no hay; por su parte en aquellas áreas donde ya se encuentren arbustos, se propone utilizarlos como plantas nodriza para establecer árboles de varias especies buscando mantener biodiversidad de especies arbóreas, en donde la teoría de la sucesión indica que el pinabete va a dominar al final. Esta propuesta ha demostrado viabilidad porque es una práctica conocida fuera de bosques naturales en áreas de San Marcos para establecer el pinabete con fines comerciales, que aprovecha la sucesión vegetal natural del área.

Palabras claves: Bosques de altura, sucesión vegetal, especie protegida, plantas nodrizas, conservación de la naturaleza.

\section{Abstract}

$\mathrm{T}$ The fir, Abies guatemalensis Rehder, is distributed in the highlands of Guatemala. According to the National Protected Areas Council (Conap), their populations are severely fragmented. For its rarity and fragmentation, it is protected by the Convention on International Trade in Endangered Species of Wild Fauna and Flora species (Cites), indicating the importance of the recovery of their forests for the proper conservation of the species. The success of his establishment may be enhanced to sow with nurse plants, which would help to survive the dry conditions of the year and frosts that occur in the higher elevations of the country during the months of November to February. This work shows the features physical and chemical and vegetation in the area of three forests of fir (A. guatemalensis) of the top of San Marcos. In addition, the proposal of ecological restoration simulating plant succession in the area. Maps were drawn, and three fir forests and surrounding described; it is shown the study the chemical composition of soil and vegetation and their representation in different ages of plant succession. Further, it is proposed the recovery of areas around patches of forest, consting of which is establish shrubby plants in open spaces where there are not yet; in areas where bushes already are, it is proposed to use it as nurse plants to establish trees of various species seeking to maintain biodiversity of tree species, where the theory of succession indicates that the fir will dominate in the end. This proposal is feasible because as it is known outside natural forests in areas of San Marcos to establish the fir for commercial purposes, which uses the natural plant succession the area.

Keywords:Upland forest, plant succession, protected species, nurse plants, nature conservation. 


\section{Introducción}

En la parte alta del departamento de San Marcos, Guatemala, hay 41 bosques de pinabete (Abies guatemalensis Rehder), que cubren un total de 1,791.39 ha, distribuidas en 11 municipios. Estos bosques tienen una extensión variable, que fluctúa entre 1.25 y 190.4 ha. Según datos del Consejo Nacional de Áreas Protegidas (Conap) y el Instituto Nacional de Bosques (Inab) (1999), el área que cubriría originalmente el pinabete a nivel nacional alcanzaba las 558,858 ha; pero para 1979 ya se reportaban únicamente 71,000 . El cambio en el uso del suelo hacia agricultura y pastoreo desde el siglo XVI, han llevado a que esta especie sea considera en peligro de extinción, y esté incluida en el Apéndice I de la lista de la Convención sobre el Comercio Internacional de Especies Amenazadas de Fauna y Flora Silvestres (Cites) desde 1997. Por tanto, urge la necesidad de recuperación de áreas de bosque para la conservación de la especie y su entorno. En primer lugar, por su valor como endémica para Mesoamérica (Jaramillo-Correa et al., 2008), y también, como la han indicado varios autores entre ellos Gómez (2004), por los servicios ecosistémicos que estos bosques prestan; por ejemplo, la regulación del ciclo hidrológico, microclima, biodiversidad, almacenamiento de dióxido de carbono, liberación de oxígeno, etcétera.

Además, aparte de la conservación de los bosques, el manejo de $A$. guatemalensis fuera de bosques presenta una gran oportunidad económica, pues se puede establecer con fines comerciales, especialmente para su venta como árbol navideño (Andersen et al., 2008; Ignosh \& Kilgore, 2005). Esto también contribuiría con la conservación al disminuir la presión de extracción ilegal de ramillas en época navideña de bosques naturales. En las áreas abiertas de los bosques se han realizado esfuerzos de reforestación con pino y pinabete por muchos años, sin embargo los resultados no han tenido el éxito deseado, debido a que durante el establecimiento de las especies arbóreas no se ha previsto la protección que deben tener durante las épocas seca y de heladas. La sucesión ecológica muestra el camino que debe seguirse en estas áreas para su recuperación, que consiste en aprovechar los arbustos que pueden servir de plantas nodrizas, en los primeros años del establecimiento de árboles (Martínez, 2013).

Para tener un mejor panorama de cómo hacerlo, uno de los pasos es el reconocimiento y caracterización del área (Vargas, 2007). Con esto se busca reconocer mejor el sistema que se quiere recuperar y sirve como base para un plan de restauración ecológica (Montenegro \& Vargas, 2008). En consecuencia, aquí se presenta la descripción general del área, las características químicas del suelo y la vegetación en tres bosques de pinabete (A. guatemalensis) de la parte alta del departamento de San Marcos, y una propuesta de restauración ecológica siguiendo la potencial ruta de la sucesión vegetal natural del área.

\section{Materiales y métodos}

El área de estudio fue en la zona de mayor elevación del departamento de San Marcos, ubicada entre los 3,000 a 3,500 m de altitud, en tres bosques y su área de influencia. Estas son: Las Nubes situado en el municipio en San José Ojetenam, Los Cuervos en Ixchiguán y Canatzaj en Tacaná, que se encuentran entre las coordenadas $15^{\circ} 10^{\prime} 03^{\prime \prime}$ a $15^{\circ} 14^{\prime} 53^{\prime \prime}$ latitud N y $91^{\circ} 17^{\prime} 18^{\prime \prime}$ y $91^{\circ} 59^{\prime} 23^{\prime \prime}$ longitud $\mathrm{O}$.

Se elaboró un mapa para cada localidad, y una descripción de las condiciones fisiográficas de cada una. Para fines de comprensión, estos bosques se encuentran en un paisaje fragmentado. Siguiendo algunos conceptos de Vila, Varga, Llausàs y Ribas (2006), se le llama fragmento o parche a la parte que aún tiene bosque, borde a las áreas de transición alrededor de los parches y las orillas a las áreas abiertas con gramíneas, plantas anuales o arbustos, que son sujetas a manejo para aumentar el área de los fragmentos actuales.

Se caracterizó la vegetación a través de la determinación de la flora en cinco etapas de sucesión; las especies fueron determinadas en el herbario Bigu de la Escuela de Biología de la Facultad de Ciencias Químicas y Farmacia de la Universidad de San Carlos de Guatemala. En los resultados se presenta el número de especies por estrato en cada etapa de sucesión. Además, se realizó una estimación comparativa del valor de importancia (IVI) de Cottam de cada etapa de sucesión integrando los tres bosques, en cuatro estratos diferentes: herbáceo inferior (0.05-0.5 m), herbáceo (0.20-1.5 $\mathrm{m})$, arbustivo (1-8 m) y arbóreo (2-60 m). El IVI es una variable sintética que se desarrolla a partir de la densidad, cobertura y frecuencia aparente de cada especie. Estas se convierten a valores reales y luego en valores relativos; se suman las tres variables y eso constituye el IVI por especie. Aquellas que tengan los valores más altos serán las más representativas del área (Matteucci \& Colma, 1982). Con esta información se elaboró una tabla resumen donde se consigna los rangos de IVI en cada etapa de sucesión y las especies que presentan los mayores valores de IVI. 
Se elaboró un perfil de vegetación de cada etapa de sucesión, donde se representa la fisonomía promedio $\mathrm{y}$ las principales especies de cada uno.

En el año 2011 se efectuó un análisis de suelo en cinco etapas de sucesión y otro en 2014 en las etapas de sucesión iniciales y con arbustos. En consecuencia, se presenta el $\mathrm{pH}$, fósforo, capacidad de intercambio catiónico (CIC), porcentaje de saturación de bases $(\% \mathrm{SB})$, porcentaje de materia orgánica $(\% \mathrm{MO})$ y nitrógeno total. Por medio de la prueba no paramétrica del test de Wilcoxon para una muestra, se realizó una prueba de significancia para cada característica química. Se calculó la media, desviación estándar, rango y coeficiente de variación para cada parámetro químico del suelo.

Finalmente, con base en las etapas de sucesión, se hace una propuesta esquemática de restauración ecológica en las áreas adyacentes a los bosques.

\section{Resultados}

\section{Caracterización descriptiva de tres bosques}

Bosque Las Nubes. El bosque Las Nubes (Figura 1), se encuentra ubicado en el municipio de San José Ojetenam, San Marcos y tiene como principal área de influencia a la aldea San Fernando y sus caseríos. Para llegar al bosque se toma la carretera nueva de Ixchiguán hacia San José Ojetenam y al empezar a descender hacia el pueblo, se cruza hacia la antigua carretera a Ixchiguán, donde se recorre cerca de un kilómetro de terracería. Tiene una extensión aproximada de 104.4 ha de acuerdo con Conap (2008). Con base en el área que la municipalidad de San José Ojetenam protege, se tienen 46.08 ha, donde se puede diferenciar fácilmente al menos tres etapas de sucesión: áreas abiertas, donde la vegetación está compuesta por plantas herbáceas, con aproximadamente 9.67 ha; área de arbustos, que en su mayoría sirven de plantas nodrizas, con aproximadamente 8.27 ha y áreas con árboles, con un área aproximada de 28.14 ha compuesta de aproximadamente de $60 \%$ de pino (Pinus pseudostrobus Lindl.) y $40 \%$ de pinabete (A. guatemalensis).

Este bosque corresponde a una hondonada que tiene dos caras, una hacia el noroccidente y la otra hacia suroriente. El rango de altitud va desde los 3,000 a los $3,460 \mathrm{~m}$. La porción entre la parte alta de la carretera nueva hacia San José Ojetenam y la carretera antigua, tiene una pendiente de $45 \%$, está desprovista de bosque y tiene una mezcla de partes totalmente abiertas y áreas con plantas arbustivas. De la carretera antigua hacia el fondo de la barranca, está lo que queda del bosque puro, aquí la pendiente aumenta y en algunos casos llega a peñascos de cerca del $90 \%$. El pastoreo de ovejas casi se ha eliminado. Sin embargo, las personas llegan a cortar la vegetación herbácea que llevan para sus animales, dañando de todas formas el crecimiento de las fases arbustivas.

En cuanto a la participación comunitaria, se infiere poca identificación y preocupación de las comunidades por este bosque, debido su participación en el cuidado del bosque y el poco interés en las reuniones realizadas, a pesar de que hay guarda recursos y participación de la municipalidad de San José Ojetenam, el deterioro es evidente, posiblemente porque no hay comunidades cercanas que se identifiquen mejor con el bosque, aunque el agua que aquí se produce sirve de abastecimiento para todas las comunidades de sus alrededores.

Bosque Los Cuervos. El bosque Los Cuervos (Figura 2), se encuentra ubicado en Ixchiguán, arriba de la población de la cabecera municipal $(3 \mathrm{~km})$, a orillas de la carretera hacia Tacaná. Con base en la información de la municipalidad de Ixchiguán, tiene una extensión aproximada de 87.86 ha, según el Conap, (2008) son 81.05 ha, de las cuales 45.96 corresponden a áreas de bosque donde la especie dominante es el pinabete y en sus bordes pino (P. pseudostrobus y P. rudis Endl.) y ciprés (Cupressus lusitanica Mill.). El área sin bosque de 39 ha, está dominada por especies herbáceas y musgos. En la época lluviosa dominan la cobertura del suelo dos especies de Alchemilla una de flor blanca y la otra lila. Cerca de 7 ha tienen plantas arbustivas que en determinado momento pueden servir como nodrizas.

La orientación principal del bosque es hacia el noroccidente, con rangos de 3,200 a 3,400 $\mathrm{msnm}$. La cara del bosque que está sin vegetación tiene una orientación principal hacia el noreste. A partir del medio día la velocidad del viento se intensifica con velocidades de hasta 15 o $16 \mathrm{~km} / \mathrm{hora}$, lo que provoca una mayor evapotranspiración de las plantas. Los esfuerzos que se han realizado por reforestar no han dado resultado y solo han quedado algunos árboles achaparrados dispersos.

En los últimos tres años ha aumentado las poblaciones de taltuzas (Orthogeomys sp.), este es un roedor subterráneo que causa daño tanto a los pastos, como a los pocos árboles achaparrados de pino dispersos del área. En 2013 se llevó a cabo una capacitación al personal de campo de la municipalidad de Ixchiguán con 


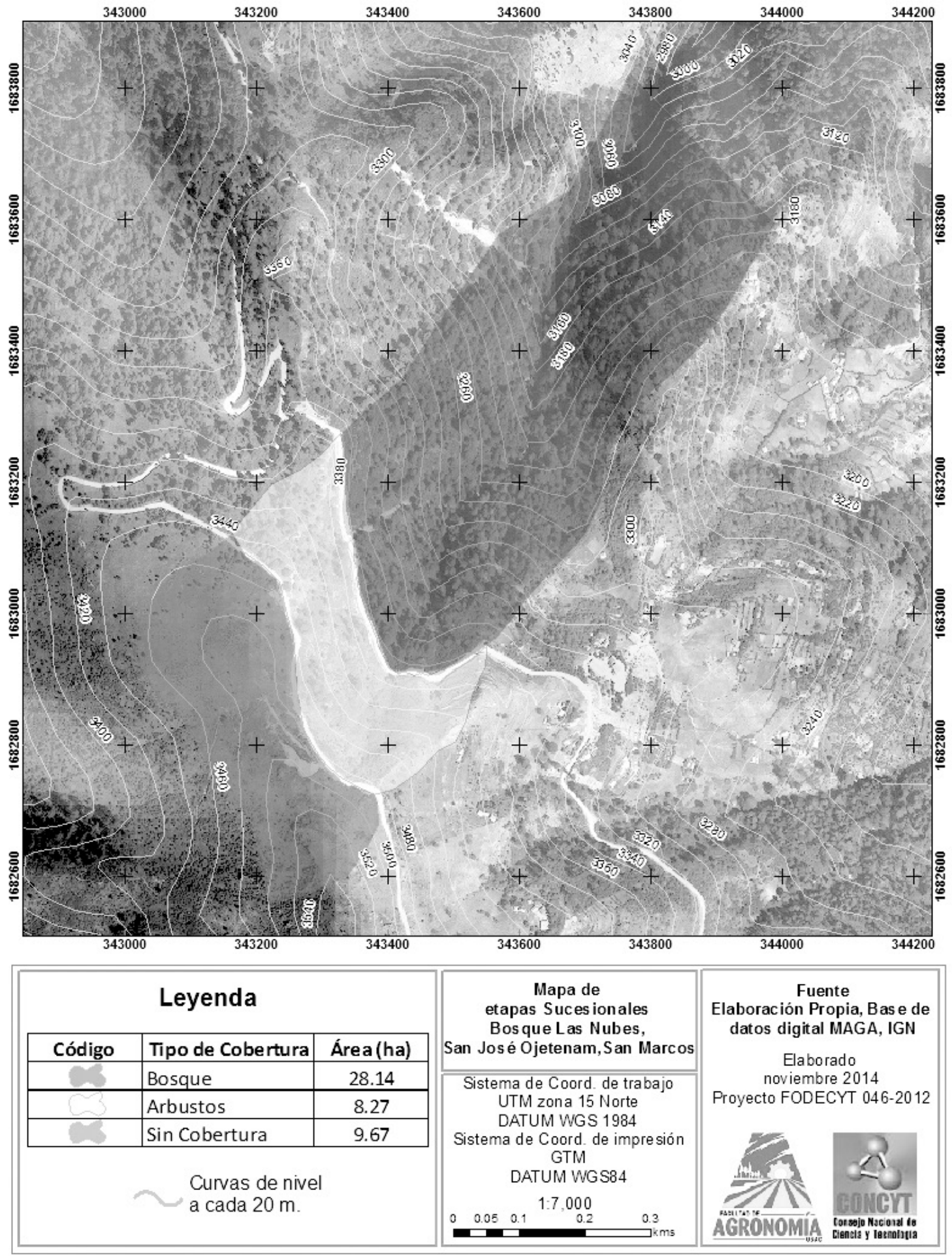

Figura 1. Mapa del bosque Las Nubes, San José Ojetenam, San Marcos. La porción oscura de arriba representa la parte con bosque y la de abajo, la que tiene arbustos y/o hierbas. 


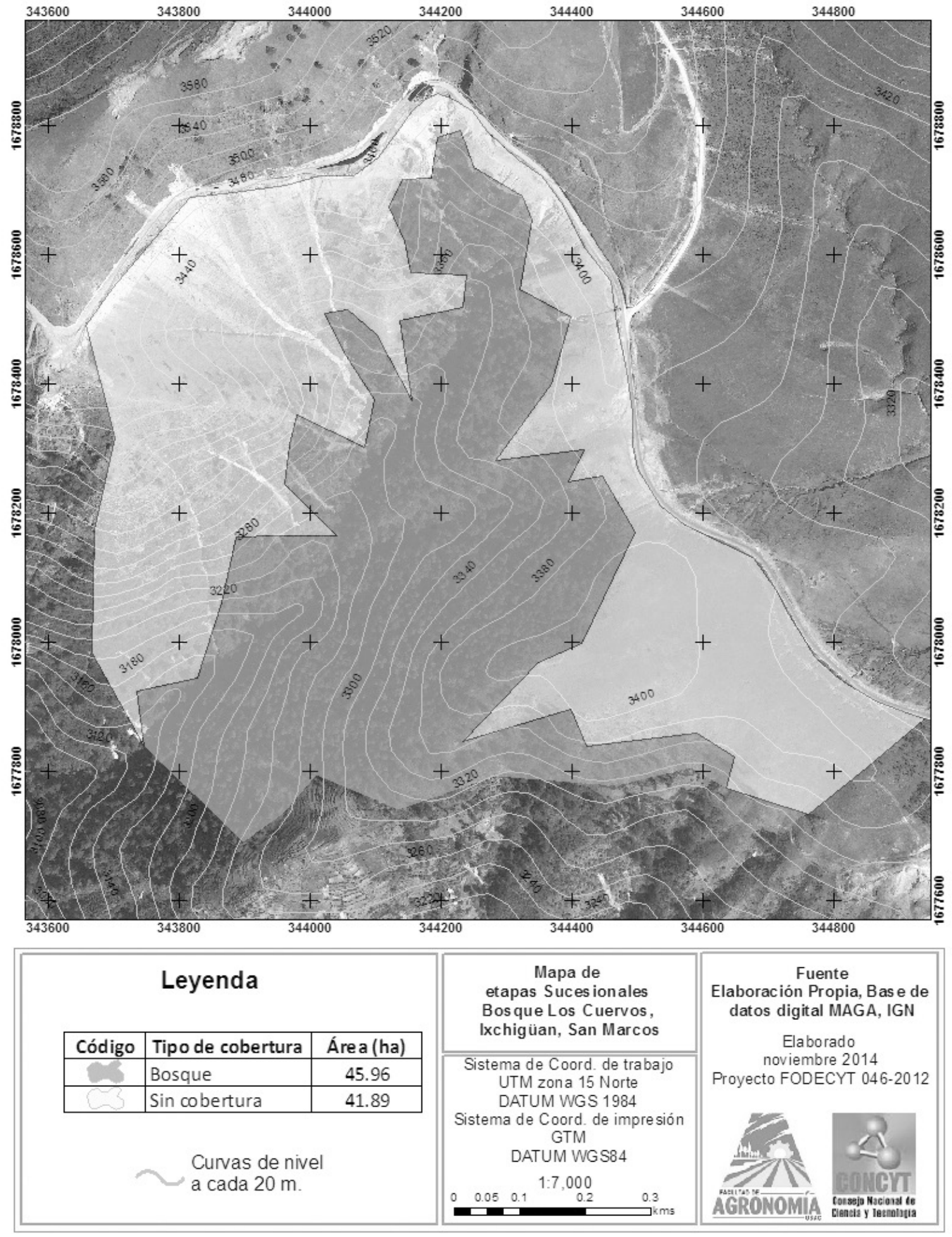

Figura 2. Mapa del bosque Los Cuervos, Ixchiguán, San Marcos, en color oscuro se presenta el área con bosque y la parte clara, el área con arbustos y hierbas. 


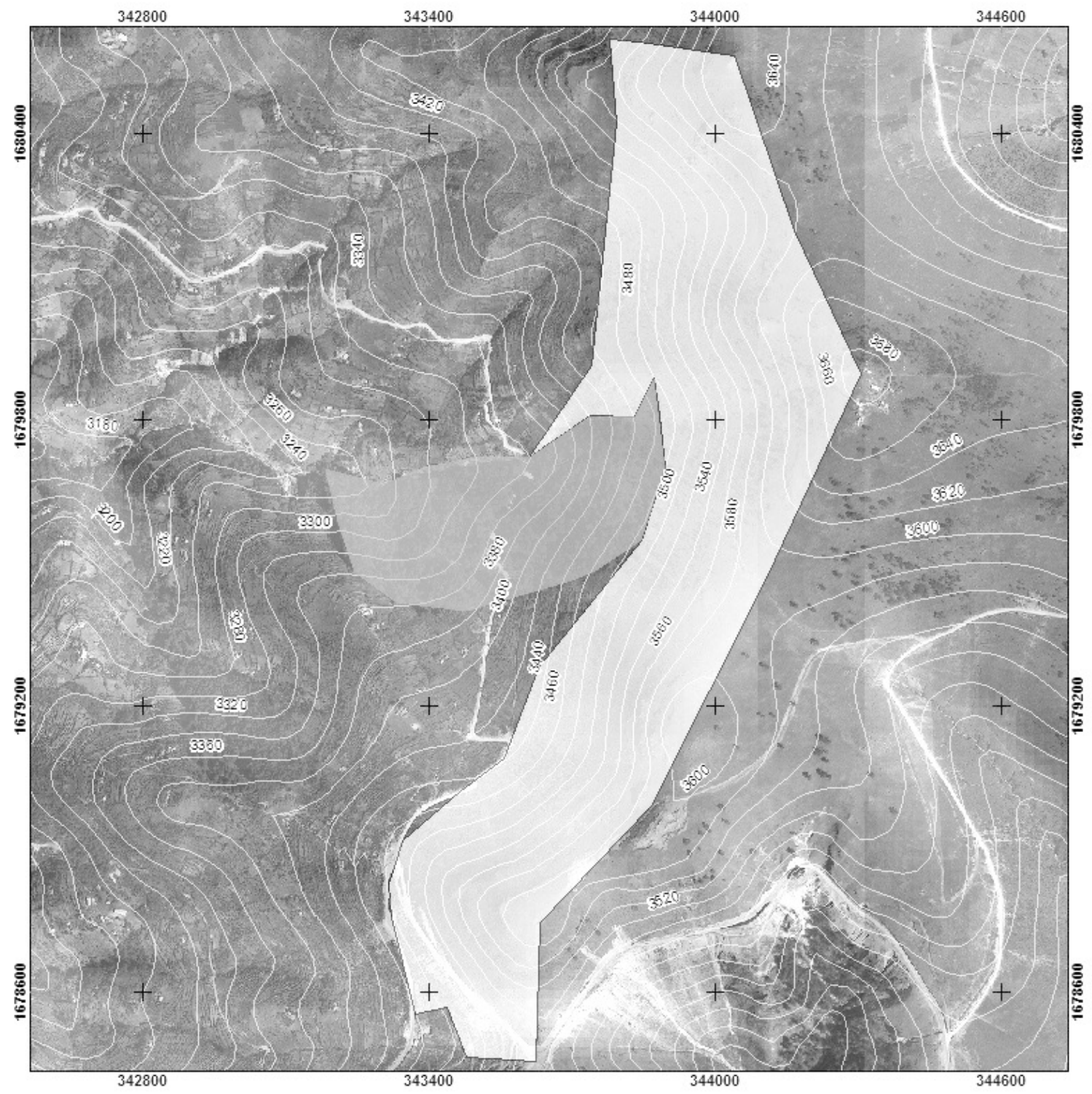

\begin{tabular}{|c|c|c|c|c|}
\hline \multicolumn{3}{|c|}{ Leyenda } & Mapa de etapas sucesioinales & \multirow{2}{*}{$\begin{array}{c}\text { Fuente } \\
\text { Elaboración Propia, Bas e de } \\
\text { datos digital MAGA, IGN } \\
\text { Elaborado }\end{array}$} \\
\hline Código & Tipo de cobertura & Área (ha) & & \\
\hline 03 & Bosque & 19.19 & \multirow{3}{*}{$\begin{array}{c}\text { Sistema de Coord. de trabajo } \\
\text { UTM zona } 15 \text { Norte } \\
\text { DATUM WGS } 1984 \\
\text { Sistema de Coord. de impresión } \\
\text { GTM } \\
\text { DATUM WGS84 }\end{array}$} & $\begin{array}{l}\text { Febrero } 2015 \\
\text { Proyecto FODECYT 046-2012 }\end{array}$ \\
\hline 3 & Sin cobertura & 85.17 & & \\
\hline \multicolumn{3}{|c|}{$\begin{array}{l}\text { Curvas de nivel } \\
\text { a cada } 20 \mathrm{~m} \text {. }\end{array}$} & & AGRONOMIA \\
\hline
\end{tabular}

Figura 3. Bosque Cantatzaj, Tacaná, San Marcos, en color más obscuro el área con bosque y en el más claro la parte con hierbas y/o arbustos. 
lo cual se logró, solo de octubre a diciembre de ese año, la captura de cerca de 20 taltuzas en cerca de 29 ha.

A pesar que el área está cercada y que los rebaños de ovejas ya no pastorean allí, hay inclusiones de ganado bovino o equino en forma esporádica, lo cual es un factor que no favorece la sucesión ecológica. Este bosque lo tiene a su cargo la municipalidad de Ixchiguán, que mantiene un constante monitoreo a través de un guarda recursos. Sin embargo, no hay comunidades locales que cuiden de este bosque, lo que hace que los esfuerzos de recuperación de la municipalidad sean difíciles, pues no hay mano de obra disponible para los trabajos a realizar.

Bosque Canatzaj. El bosque Canatzaj (Figura 3), se encuentra ubicado en Tacaná, donde finaliza el cerro Cotzic, por lo tanto colinda con Ixchiguán. Su área de influencia es principalmente para 10 comunidades, entre ellas Flor de Mayo, Sanabaja, Santa María, Laureles y Canatzaj. Para llegar al bosque, se toma por la carretera asfaltada de Ixchiguán hacia Tacaná, $6 \mathrm{~km}$ después de Ixchiguán, se cruza hacia la derecha a un camino de terracería, donde se empieza a descender y a $1.5 \mathrm{~km}$ empieza el bosque. Tiene un área total de 111.27 ha, de las cuales solo 19.40 ha tienen bosque principalmente de pinabete, esta última área es la que reporta el Conap, (2008). En algunas áreas se ha realizado reforestación con ciprés y también en los bordes del bosque se puede encontrar pino. Gran parte del área está sin cobertura forestal que corresponde a 91.87 ha, de las cuales cerca del $85 \%$ son áreas cubiertas por gramíneas y algunas plantas anuales y en el 15\% restante hay presencia de arbustos. La orientación de la pendiente de la porción sin bosque, tiene una parte hacia el sur y otra hacia el norte y la porción con bosque tiene una parte hacia el noroccidente, otra hacia el norte y una más hacia el sur. El rango altitudinal varía entre 3,000 a 3,500 msnm.
Este bosque tiene influencia en 10 comunidades por los nacimientos de agua que hay allí. Posee guarda recursos que monitorean constantemente el área para evitar el pastoreo de ovejas y extracciones ilegales. Cada año los comunitarios procuran establecer árboles. La organización social de estas comunidades, es bastante robusta, pues sus comités de agua procuran velar por la protección del bosque, anualmente se elige una nueva junta directiva que dirige las acciones. Esto provoca que lo que aún queda de bosque refleje el cuidado que se hace del mismo.

\section{Análisis de la vegetación}

En total se reportan 71 especies de plantas; en la Tabla 1 se presenta el número de especies por estrato en cada etapa de sucesión. En todas las etapas los estratos de vegetación herbáceo inferior y herbáceo superior, tiene un similar número de especies. En los etapas 4 y 5 que están dominados por árboles, los demás estratos siguen manteniendo casi igual número de especies, que en las etapas abiertas, lo que varía es la densidad de cada especie. En los etapas 1 y 2, no hay presencia de árboles, en el etapa 3 es donde mayor cantidad de especies arbóreas hay y disminuye a una en el etapa 5. Se nota un incremento de especies del etapa 1 al 3 y luego una disminución hacia el etapa 5.

En el cálculo del IVI calculado para cada especie, se encontró una amplia distribución de valores, es decir no hay especies dominantes a excepción de las etapas donde hay árboles (Tabla 2). Se puede visualizar que en el caso del estrato herbáceo inferior hay cinco especies con mayor valor de IVI de las cuales cuatro son musgos, que tienen presencia en las cinco etapas, la especie Hypnum amabilie (Mitt.) Hampe está presente en las cinco etapas. En el estrato herbáceo superior hay 11

Tabla 1

Resumen de número de especies por estrato y etapa de sucesión en los tres bosques estudiados.

\begin{tabular}{lccccc}
\hline & \multicolumn{5}{c}{ Número de especies por etapa de sucesión } \\
\cline { 2 - 6 } Estrato & 1 & 2 & 3 & 4 & 5 \\
\hline Herbáceo inferior & 11 & 10 & 12 & 12 & 13 \\
Herbáceo superior & 14 & 18 & 16 & 14 & 16 \\
Arbustivo & 7 & 18 & 17 & 18 & 18 \\
Árboles & & & 4 & 4 & 1 \\
Total & 32 & 46 & 53 & 48 & 48 \\
\hline
\end{tabular}


Tabla 2

Especies con mayor valor de importancia a través de cinco etapas de sucesión de tres bosques de pinabete (A. guatemalensis) de San Marcos, Guatemala

\begin{tabular}{|c|c|c|c|c|c|}
\hline & Etapa 1 & Etapa 2 & Etapa 3 & Etapa 4 & Etapa 5 \\
\hline \multicolumn{6}{|l|}{ Estrato herbáceo inferior } \\
\hline Rango de IVI & $8.81-89.46$ & $6.92-88.95$ & $6.39-91.21$ & $6.80-71.38$ & $4.74-103.82$ \\
\hline $\begin{array}{l}\text { Brachypodium mexicanum (Roem. \& } \\
\text { Schult.) Link }\end{array}$ & 50.42 & & & & \\
\hline Geranium repens H.E. Moore & & 59.21 & & & \\
\hline Hypnum amabilie (Mitt.) Hampe & 87.46 & 88.95 & 91.21 & 61.25 & 103.82 \\
\hline $\begin{array}{l}\text { Vesicularia vesicularis (Schwägr.) } \\
\text { Broth. var. portoricensis (Brid.) Buck. }\end{array}$ & 60.16 & & & 71.38 & \\
\hline $\begin{array}{l}\text { Hypopterygium tamariscinum (Hedw.) } \\
\text { Brid. }\end{array}$ & & & 44.84 & & 65.57 \\
\hline \multicolumn{6}{|l|}{ Estrato herbáceo superior } \\
\hline Rango de IVI & $6.73-53.57$ & $4.64-60.36$ & $5.50-44.74$ & $6.77-42.46$ & $6.10-45.26$ \\
\hline Salvia sp. L. & 53.57 & 29.17 & & & \\
\hline $\begin{array}{l}\text { Eupatorium liebmannii Sch. Bip. ex } \\
\text { Klatt }\end{array}$ & 36.97 & & & & \\
\hline Bidens ostruthioides (DC.) Sch. Bip. & 25.06 & 60.36 & 44.17 & 42.46 & 45.26 \\
\hline Cirsium radians Benth. & & 47.72 & & & \\
\hline Hackelia skutchii I.M. Johnst. & & 26.7 & 31.08 & & \\
\hline Eryngium cymosum F. Dolaroche & & & 44.74 & 37.04 & \\
\hline $\begin{array}{l}\text { Muhlenbergia macroura (Kunth) } \\
\text { Hitchc. }\end{array}$ & & & 35.46 & & \\
\hline Lopezia hirsuta Jacq. & & & & 34.02 & 40.17 \\
\hline Lamouxouxia xalapensis Benth. & & & & 37.57 & \\
\hline Bomarea hirtella (Kunth) Herb. & & & & & 41.78 \\
\hline \multicolumn{6}{|l|}{ Estrato arbustivo } \\
\hline Rango de IVI & $16.36-89.60$ & $4.19-53.36$ & $5.00-73.25$ & $4.57-55.54$ & $4.21-47.08$ \\
\hline Acaena elongata $\mathrm{L}$. & 84.37 & & 73.25 & 26.34 & 47.08 \\
\hline Salvia gracilis Benth. & 89.6 & & & & \\
\hline Verbesina hypoglauca Sch. Bip. ex Klatt & & 29.82 & & & \\
\hline Symphoricarpos microphyllus Kunth & & 31.24 & & & \\
\hline Rubus trilobus L. & & 30.54 & & & \\
\hline Lupinus ehrenbergii Schltdl. & & 53.36 & 47.22 & 35.93 & 31.85 \\
\hline Baccharis vaccinioides Kunth & & & 35.38 & 22.23 & \\
\hline $\begin{array}{l}\text { Salvia cinnabarina M. Martens \& } \\
\text { Galeotti }\end{array}$ & & & & 55.54 & \\
\hline
\end{tabular}


Tabla 2, continuación

\begin{tabular}{|c|c|c|c|c|c|}
\hline & Etapa 1 & Etapa 2 & Etapa 3 & Etapa 4 & Etapa 5 \\
\hline Gaultheria sp. L. & & & & & 33.82 \\
\hline \multicolumn{6}{|l|}{ Estrato de árboles } \\
\hline Rango de IVI & & & $\begin{array}{l}12.63- \\
102.11\end{array}$ & $28.15-119.50$ & $300-300$ \\
\hline Abies guatemalensis Rehder & & & 52.39 & 119.19 & 300 \\
\hline Pinus ayacahuite C. Ehrenb. ex Schltdl. & & & 53.16 & & \\
\hline $\begin{array}{l}\text { Neocupressus lusitanica (Mill.) de } \\
\text { Laub. }\end{array}$ & & & 102.11 & 119.5 & \\
\hline
\end{tabular}

especies con mayor valor de IVI; la especie Bidens ostruthioides (DC.) Sch. Bip. es común en estos mismos. En el estrato de arbustos se encontraron nueve especies con mayor valor de IVI, de las cuales Acaena elongata L. es común en las cinco etapas. Es interesante ver que Lupinus ehrenbergii Schltdl., Baccharis vaccinioides Kunth, Salvia cinnabarina M. Martens \& Galeotti y Gaultheria sp. L. tienen mayor valor de importancia en las etapas con árboles. En el estrato de árboles solo hay tres especies que dominan y en la etapa cinco únicamente se encuentra al pinabete (A. guatemalensis).

\section{Descripción de las etapas de sucesión vegetal}

A partir de la vegetación encontrada se prepararon cinco perfiles con la escala de acuerdo a la altura de las plantas de los estratos dominantes. En las figuras de la 4 a 8 se muestra la fisonomía promedio que presentan las etapas de sucesión vegetal.

La fisonomía de la etapa 1 se presenta en la Figura 4. Es producto de áreas que anteriormente fueron utilizadas para pastoreo de ovejas, tienen de 9 a 12 años de estar excluidas de pastoreo, ya que las comunidades y municipalidad las cuidan para proteger y conservar a $A$. guatemalensis. El aspecto general es de un área abierta, con altura de plantas hasta $1.25 \mathrm{~m}$, el estrato herbáceo inferior está dominado por musgos que cubren el suelo todo el año. Luego el estrato superior con especies herbáceas anuales o semi perennes. Se empieza a notar presencia de arbustos jóvenes dispersos.

La etapa 2, se presenta en la Figura 5, la vegetación tiene edades entre 12 a 20 años y la fisonomía es dominada por arbustos, en especial Rubus trilobus L., $B$. vaccinioides y A. elongata.

En esta etapa las especies del estrato inferior como musgos y otras especies, sobreviven mejor a lo largo del año a causa de la sombra y humedad que proporcionan los arbustos. Esta etapa sucesional es propicia para la regeneración natural, también se nota el aporte de hojas al suelo por parte de los arbustos.

En la Figura 6, se representa la etapa 3 el cual tiene una edad aproximada de 20 a 45 años. La fisonomía es dominada por arbustos y árboles. Esta etapa presenta la mayor riqueza de árboles, que consiste en seis especies tanto de plantas de hoja ancha como angosta. También es más probable encontrar regeneración natural en comparación con las dos primeras etapas de sucesión.

La etapa 4 se presenta en la Figura 7, según los pobladores locales tiene una edad aproximada de 45 a 65 años. A. guatemalensis empieza a ser dominante sobre las otras especies arbóreas. El estrato de arbustos a pesar de ser diverso, disminuye en cobertura comparado con las etapas anteriores, se puede notar más suelo descubierto. Aunque con menor cobertura, el estrato herbáceo inferior se mantiene durante todo el año y aquí el papel de los musgos en la liberación del agua se hace más evidente, pues se puede ver como las gotas de agua se van liberando lentamente hacia el suelo.

En la Figura 8, se tiene un esquema de la etapa 5, constituida por los núcleos de bosque puro de pinabete. Tiene una edad de 85 a 200 años según los pobladores locales, está compuesto por árboles de diferentes edades, los más antiguos son sobrevivientes de la extracción selectiva que se realizó en el pasado, lo que hoy se puede corroborar al ver su fisonomía. Por la apariencia, se estimó que sólo un $20 \%$ de los árboles sobrepasan los 200 años. La densidad de árboles y el hecho que hubo pastoreo en el sotobosque permiten que arbustos $\mathrm{y}$ hierbas sigan creciendo en el interior. 

dium mexicanum 4. Houstonia serpyllacea, 5. Vesicularia vesicularis var. portoricensis, 6. Hypnum amabilie y 7. Weldenia candida. Estrato herbáceo superior 8. Bidens ostruthioides, 9. Cirsium radians, 10. Eryngium cymosum, 11. Hackelia skutchii, 12. Piptochaetium sp., 13. Salvia sp., 14 . Senecio callosus, 15. Ranunculus geoides, 16. Stevia incognita. Estrato de arbustos 17. Acaena elongata, 18. Baccharis vaccinioides, 19. Lupinus ehrenbergii, 20. Rubus trilobus, 21. Symphoricarpos microphyllus, 22. Salvia cinnabarina y 23. Salvia gracilis. 


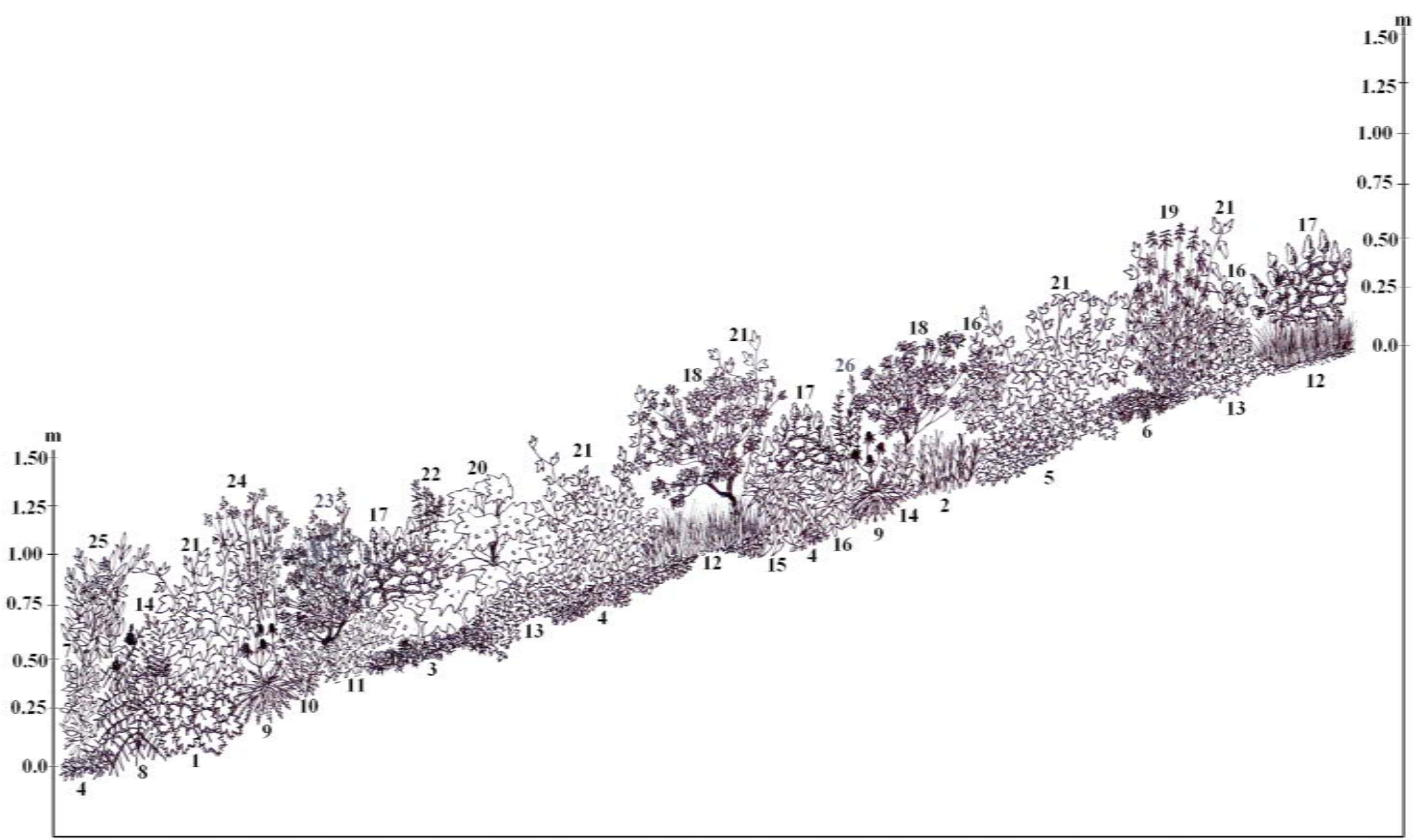

Figura 5. Etapa 2, vegetación alrededor de bosques de pinabete. Estrato herbáceo inferior 1. Alchemilla pectinata, 2. Brachypodium mexicanum, 3. Vesicularia vesicularis var. portoricensis, 4. Houstonia serpyllacea, 5. Geranium repens y 6. Hypnum amabilie. Estrato herbáceo superior 7. Bidens ostruthioides, 8. Cirsium radians, 9. Eryngium cymosum, 10. Hackelia skutchii, 11. Lopezia hirsuta, 12. Piptochaetium sp., 13. Ranunculus geoides, 14. Salvia sp., 15. Senecio callosus y 16. Stevia incognita. Estrato de arbustos 17. Acaena elongata, 18. Baccharis vaccinioides, 19. Lupinus ehrenbergii, 20. Roldana heterogama, 21. Rubus trilobus, 22. Stevia polycephala, 23. Symphoricarpos microphyllus, 24. Verbesina apleura, 25. Salvia cinnabarina y 26. Salvia gracilis. 


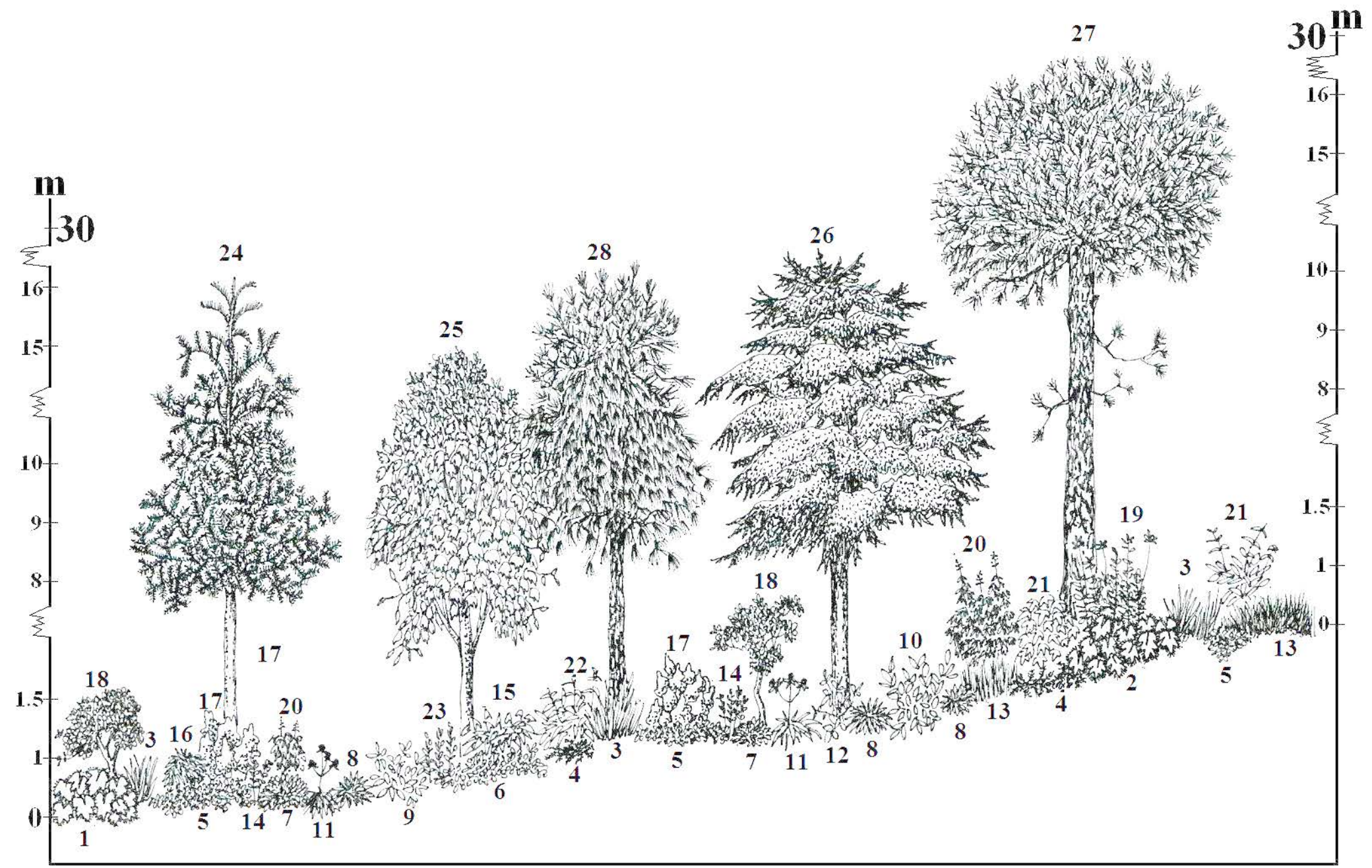

Figura 6. Etapa 3, vegetación alrededor bosques de pinabete. Estrato herbáceo inferior 1. Alchemilla pectinata, 2. Alchemilla guatemalensis, 3. Brachypodium mexicanum, 4. Vesicularia vesicularis var. portoricensis, 5. Houstonia serpyllacea, 6. Sematophyllum galipense, 7. Hypnum amabilie y 8. Weldenia candida. Estrato herbáceo superior 9. Bidens ostruthioides, 10. Bidens sp., 11. Eryngium cymosum, 12. Lopezia hirsuta, 13. Muhlenbergia macroura, 14. Salvia sp., 15. Senecio callosus y 16. Sigesbeckia jorullensis. Estrato de arbustos 17. Acaena elongata, 18. Baccharis vaccinioides, 19. Fuchsia striolata, 20. Lupinus ehrenbergii, 21. Rubus trilobus, 22. Salvia cinnabarina y 23. Salvia gracilis. Estrato de árboles 24. Abies guatemalensis, 25. Alnus acuminata, 26. Neocupressus lusitanica, 27. Pinus ayacahuite y 28. Pinus rudis. 


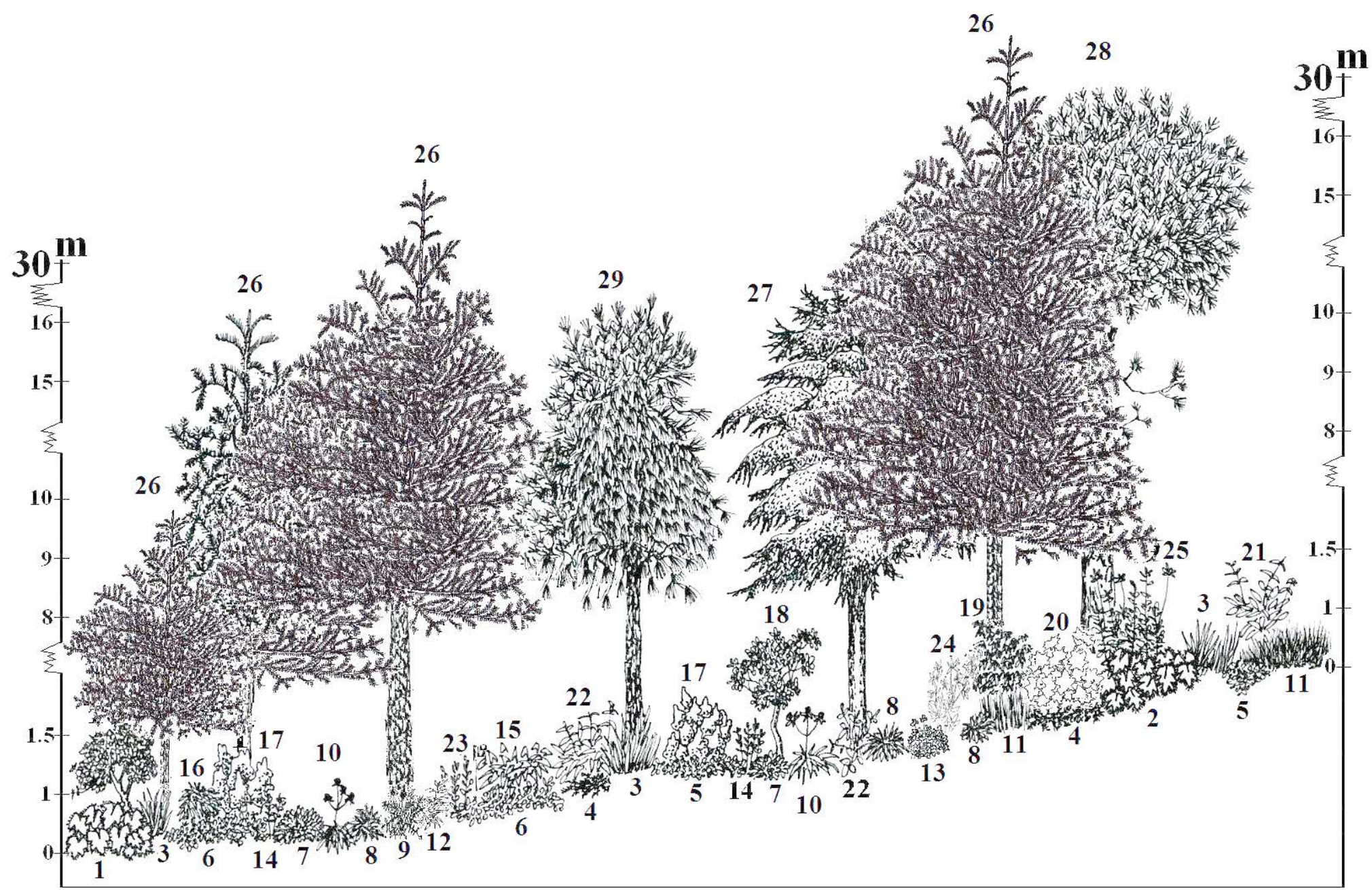

Figura 7. Etapa 4, vegetación alrededor bosques de pinabete. Estrato herbáceo inferior 1. Alchemilla pectinata, 2. Alchemilla guatemalensis, 3. Brachypodium mexicanum, 4. Vesicularia vesicularis var. portoricensis, 5. Houstonia serpyllacea, 6. Sematophyllum galipense, 7. Hypnum amabilie y 8. Weldenia candida. Estrato herbáceo superior 9. Cirsium radians, 10. Eryngium cymosum, 11. Muhlenbergia macroura, 12. Lopezia hirsuta, 13. Ranunculus geoides 14. Salvia sp., 15. Senecio callosus y 16. Sigesbeckia jorullensis. Estrato de arbustos 17. Acaena elongata, 18. Baccharis vaccinioides, 19. Fuchsia striolata, 20. Roldana heterogama, 21. Salvia cinnabarina, 22. Salvia gracilis, 23. Stevia polycephala, 24. Verbesina apleura y 25. Verbesina hypoglauca. Estrato de árboles 26. Abies guatemalensis, 27. Neocupressus lusitanica, 28. Pinus ayacahuite y 29. Pinus rudis. 


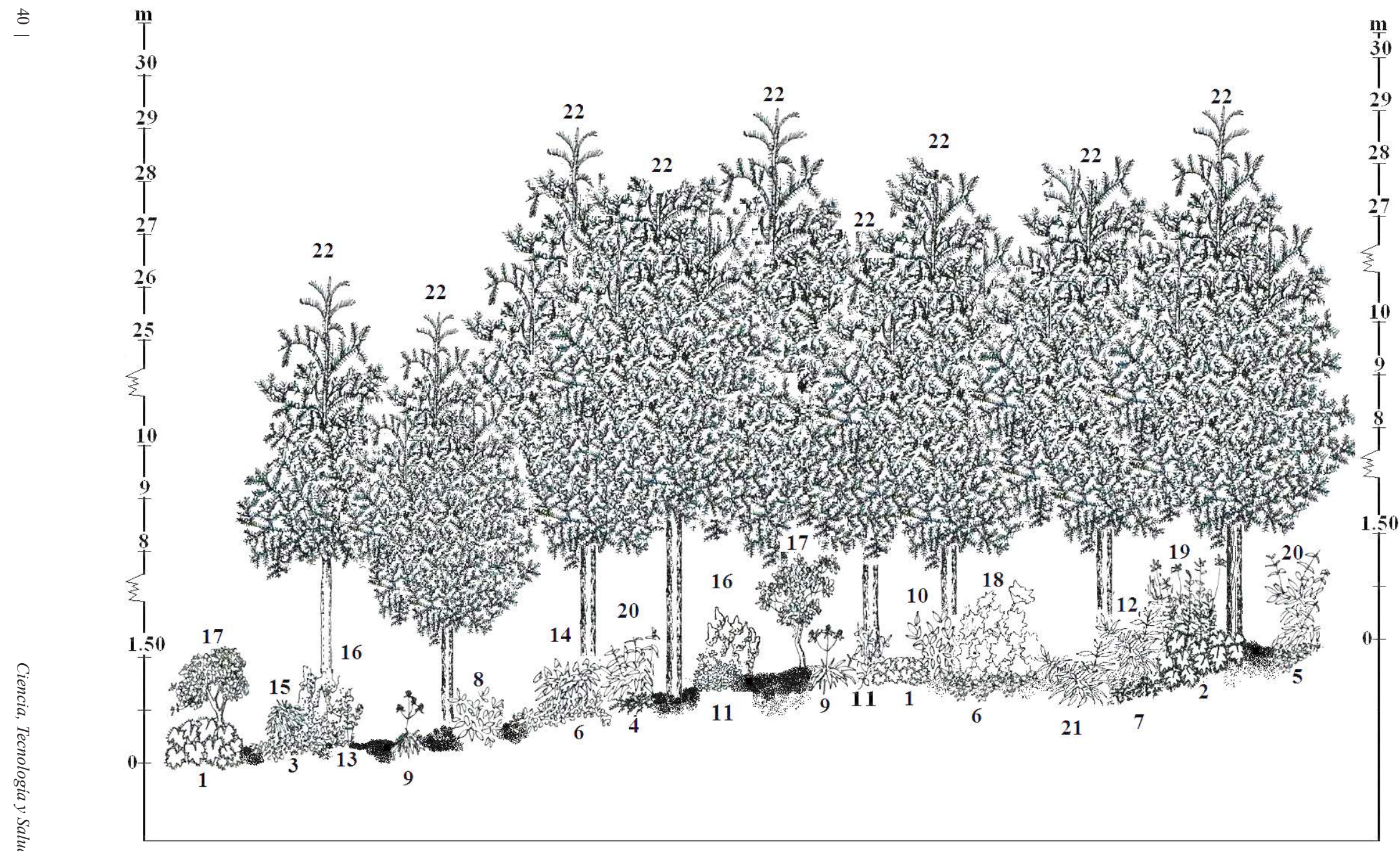

Figura 8. Etapa 5, vegetación de bosques de pinabete. Estrato herbáceo inferior 1. Alchemilla pectinata, 2. Alchemilla guatemalensis, 3. Vesicularia vesicularis var. portoricensis, 4. Sematophyllum galipense, y 5. Hypnum amabilie. Estrato herbáceo superior 6. Bidens ostruthioides, 7. Bidens sp., 8. Eryngium cymosum, 9. Hackelia skutchii, 10. Lopezia hirsuta, 11. Ranunculus geoides, 12. Salvia sp., 13. Senecio callosus y 14. Sigesbeckia jorullensis. Estrato de arbustos 15. Acaena elongata, 16. Baccharis vaccinioides, 17. Fuchsia striolata, 18. Roldana heterogama, 19. Salvia cinnabarina, 20. Stevia polycephala y 21. Verbesina hypoglauca. Estrato de árboles 22. Abies guatemalensis 


\section{Análisis de suelos}

Los resultados del análisis de suelo para los tres bosques muestran que el $\mathrm{pH}$ es ligeramente ácido, y que en promedio no sobrepasa el valor de 6 (Tabla 3). El fósforo es un elemento cuyos valores están por debajo del rango óptimo que es de 12 a 16 ppm, con alta variación en los valores promedio. La capacidad de intercambio catiónico (CIC) tiene valores y rangos similares en los tres bosques. El \% SB se encuentra en rangos amplios que van de menos de 25 hasta $59 \%$ lo que se comprueba con los altos valores de varianza y coeficiente de variación. El \%MO se encuentra en un rango de 6 a $32 \%$ está por arriba del mínimo de $5 \%$ para los suelos agrícolas. El porcentaje de nitrógeno total $(\% \mathrm{NT})$ tiene valores similares para los tres bosques estudiados. Como puede notarse en la última columna no hay diferencia significativa en el contenido de los parámetros estudiados del suelo en los tres bosques a través de las diferentes etapas de sucesión.

Tabla 3

Información del análisis de suelo de tres bosques de pinabete.

\begin{tabular}{|c|c|c|c|c|c|}
\hline Sitio & $\begin{array}{l}\text { Parámetro } \\
\text { P (ppm) }\end{array}$ & Media \pm DS & Rango & C.V. & Sig. \\
\hline \multicolumn{6}{|c|}{ Canatzaj } \\
\hline & $\mathrm{pH}$ & $5.78 \pm 0.6242$ & $4.90-6.80$ & 10.8 & N.S. \\
\hline & $\mathrm{P}(\mathrm{ppm})$ & $5.12 \pm 3.50$ & $2.78-15.18$ & 72.25 & N.S. \\
\hline & CIC (Meq/ $100 \mathrm{~g}$ ) & $44.50 \pm 13.16$ & $22.61-69.12$ & 29.56 & N.S. \\
\hline & SB (\%) & $28.02 \pm 18.14$ & $3.61-60.56$ & 64.76 & N.S. \\
\hline & M.O (\%) & $17.10 \pm 5.14$ & $7.32-26.63$ & 30.06 & N.S. \\
\hline & N Total (\%) & $0.7609 \pm 0.2409$ & $0.39-1.26$ & 31.66 & N.S. \\
\hline \multicolumn{6}{|c|}{ Los Cuervos } \\
\hline & $\mathrm{pH}$ & $5.73 \pm 0.5658$ & 4.9-7-0 & 9.88 & N.S. \\
\hline & $\mathrm{P}(\mathrm{ppm})$ & $5.72 \pm 3.07$ & $2.56-12.21$ & 53.71 & N.S. \\
\hline & CIC (Meq/ 100 g) & $41.10 \pm 13.08$ & $25.42-49.56$ & 31.82 & N.S. \\
\hline & SB (\%) & $23.59 \pm 16.92$ & $2.9-59.46$ & 71.76 & N.S. \\
\hline & M.O (\%) & $16.68 \pm 6.60$ & $6.06-27.29$ & 39.59 & N.S. \\
\hline & N Total (\%) & $0.7554 \pm 0.3020$ & $0.39-1.34$ & 39.98 & N.S. \\
\hline \multicolumn{6}{|c|}{ Las Nubes } \\
\hline & $\mathrm{pH}$ & $5.3 \pm 0.4390$ & $4.6-5.8$ & 8.28 & N.S \\
\hline & $\mathrm{P}(\mathrm{ppm})$ & $9.70 \pm 11.48$ & $2.72-34.24$ & 118.36 & N.S \\
\hline & CIC (Meq/ $100 \mathrm{~g}$ ) & $45.02 \pm 10.73$ & $25.72-61.74$ & 23.84 & N.S \\
\hline & SB (\%) & $25.96 \pm 18.43$ & $1.89-56.55$ & 70.97 & N.S \\
\hline & M.O (\%) & $17.74 \pm 9.45$ & $6.39-31.96$ & 53.26 & N.S \\
\hline & N Total (\%) & $0.7508 \pm 0.3432$ & $0.34-1.30$ & 45.72 & N.S \\
\hline
\end{tabular}




\section{Consideraciones para la recuperación del área boscosa por medio de restauración ecológica}

La Figura 9 muestra de forma esquemática del lado izquierdo, como está el bosque actualmente: (a) con áreas abiertas con musgos, gramíneas, anuales y algunos arbustos; (b) más cercano al área de árboles está una faja con dominancia de arbustos; y (c) los parches de bosque. Del lado derecho se representa, el mismo esquema, mostrando los trabajos de restauración que se propone realizar: en las áreas abiertas se han establecido arbustos, que en años posteriores sirven como plantas nodrizas para establecer árboles de varias especies, con mayor proporción de pinabete. En las áreas donde ya hay arbustos, se muestra que se han sembrado especies de árboles (pino, encino, aliso y pinabete) que provienen de vivero. Este proceso si se realiza cada año y con el debido seguimiento y evaluación, puede permitir en 15 a 20 años una recuperación sustancial de estos bosques.

\section{Discusión}

El presente estudio es un ejemplo de lo que pasa en los demás bosques de la parte alta de San Marcos; donde hay una degradación histórica de las áreas originales, al cederse áreas forestales para agricultura y pastoreo de ovejas, lo cual está asentado en los anales de historia de la época colonial (Gordillo, Muñoz, \& Estrada, 2005), que muestran que el número de cabezas de ganado ovino en el área llegó a alcanzar hasta 2 mi-

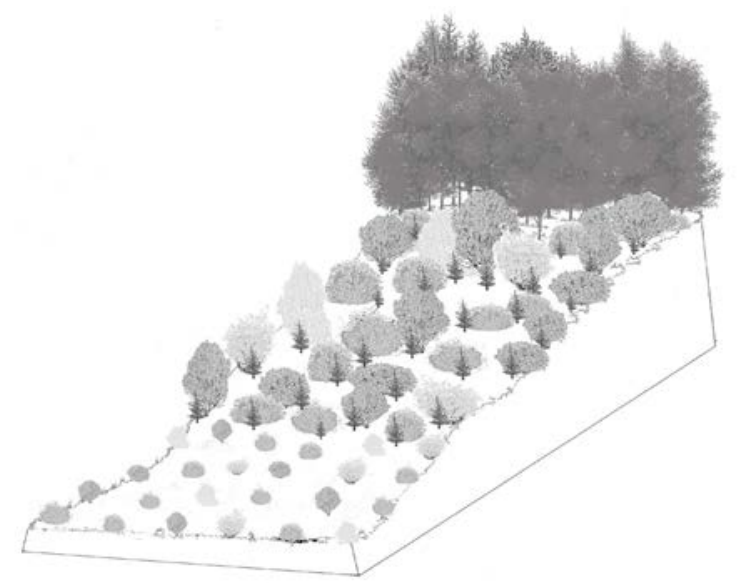

llones en el siglo XIX (MacLeod, 1980). También en las toponimias se nota el uso de la tierra, municipios como Ixchiguán, cuyo nombre se deriva de la planta ixchigua al parecer apetecida por las ovejas, pues el área fue ocupada originalmente para la crianza de este ganado (Gall, 1999, p. 336), cabe anotar que los pobladores locales y guarda recursos no saben a cuál planta pertenece este nombre. Una situación importante y que posiblemente ha contribuido a la conservación, es que actualmente ya no se pastorea ganado ovino; además las municipalidades y comunidades tienen una vigilancia constante.

En la degradación de los ecosistemas, las partes más afectadas son la vegetación y el suelo. La vegetación porque es la porción más evidente, especialmente en ecosistemas de bosque donde la fisonomía cambia drásticamente cuando los árboles son sustituidos por pastizales o cultivos (Meneses-Tovar, 2011). Por otro lado, el suelo cuyo daño inicial es menos visual, trae como producto el desbalance de ciclos biogeoquímicos, donde juega un papel importante la vida microbiana. Con esto, los minerales también se ven afectados al grado de llegar a suelos relativamente pobres en vida y en minerales aprovechables. El grado de deterioro más evidente del suelo es cuando la erosión hídrica forma cárcavas que provocan la pérdida total de los horizontes superficiales que les ha llevado cientos de años formarse (Comisión Nacional Forestal, 2013). En las orillas de los bosques estudiados, en áreas puntuales, se puede notar alguna formación de cárcavas, pero en su mayoría el efecto aún no ha llegado a este extremo, lo que puede atribuirse a que un alto porcentaje de las áreas, a pesar de ser abiertas, tienen musgos que retie-

Figura 9. Esquema de área junto a bosque de pinabete sujeto a restauración ecológica. 
nen agua y evitan la erosión acelerada, como ya fue señalado por Martínez (2013).

Con respecto a la condición química de los suelos estudiados, cabe señalar que no hay diferencia significativa en sus contenidos, sin importar la etapa de sucesión, de donde puede interpretarse que en las áreas abiertas la pérdida de suelo no ha sido extrema, situación positiva, pues esto puede ayudar mejor en la recuperación de la vegetación. Las cantidades de fósforo en la mayoría de los casos está por debajo de los mínimos, por lo que se plantea que la aplicación de abonos fosfatados debe ser considerado en el establecimiento de árboles, porque esto puede ser un factor limitante, dado que su presencia en cantidades adecuadas ayuda a los procesos fisiológicos en los que participa. Además facilita las relaciones mutualistas de microorganismos y raíces (Segura, Castillo, \& Alvarado, 2006). En la CIC los valores pueden considerar de altos a muy altos (Fassbender, 1975), lo que significa que hay una adsorción de cationes, influenciada principalmente por el contenido de materia orgánica (Kass, 1998), a pesar de que la textura de estos suelos es franco arenosa y los valores de $\mathrm{pH}$ ácido, teóricamente debería influir para que los valores de CIC fueran más bajos.

El \%SB, muestra valores relativamente bajos (< $50 \%$ ) lo que indica que las posiciones de cambio están ocupadas principalmente por $\mathrm{H}^{+} \mathrm{y} \mathrm{Al}^{+++}$, que representan la acidez intercambiable (Fassbender, 1975), esto es corroborado por los valores ácidos de $\mathrm{pH}$. Por considerarse suelos pobres en nutrientes (Kass, 1998), en el manejo de estos suelos, la corrección del $\mathrm{pH}$ puede contribuir a dar una respuesta positiva a la aplicación de enmiendas, principalmente de nitrógeno y fósforo.

$\mathrm{El} \% \mathrm{MO}$ se presenta por arriba del promedio óptimo (5\% se establece para suelos agrícolas) en todos las etapas de sucesión, lo que está acorde a lo señalado por Fassbender (1975) que indica que el contenido de materia orgánica es mayor de acuerdo con la altitud y precipitación. Sin embargo, el grado de descomposición se supone bajo, lo que es característico de los ambientes de altitud y frío, en donde aunque exista descomposición de los materiales vegetales, la mineralización es bastante lenta por factores como el $\mathrm{pH}$ y la temperatura. Por lo que un manejo durante la restauración ecológica es acelerar esta descomposición, para hacer disponible los nutrientes contenidos en ella.

El pinabete se comporta como semiperennifolio, siempre verde, aunque no se cuantificó, se estima que su aporte en hojarasca al suelo es bajo, posiblemente es otra razón que permite el crecimiento de plantas her- báceas en el sotobosque, pues el suelo no se acidifica más allá de $\mathrm{pH}$ de 5.5 .

En relación a vegetación, se puede notar que la mayoría de especies están presentes en todas las etapas de sucesión, lo que muestra el grado de intervención que han tenido estas áreas, donde el pastoreo ha facilitado su presencia, incluso en el propio bosque. Los esquemas presentados muestran la ruta de sucesión que siguen estás áreas, y que señalan el camino que debe seguirse su recuperación. Sin embargo, en esta zona la sucesión ecológica es muy lenta y como lo ha indicado Martínez (2013) muchas etapas están en una fase detenida, que necesita dinamizarse a partir de acciones de manejo ya que el disturbio ha llevado a que los ecosistemas pierdan gran parte de su capacidad de recuperación natural.

De acuerdo con la intención de conservación de estas áreas, con la recuperación se quisiera llevar el ecosistema hacia buscar el retorno de cómo era originalmente según la teoría inicial de la restauración ecológica (Bradshaw, 2002), sin embargo tomando en consideración varias razones prácticas esto se ha quedado solo en la teoría, posiblemente inalcanzable, así Hobbs y colaboradores (2014) discuten sobre una gama de escenarios los cuales se pueden alcanzar por medio de la restauración y proponen que la tendencia es a crear ecosistemas híbridos y nuevos, que ofrezcan los bienes y servicios que las poblaciones humanas requieren.

Aunque es cierto que los bosques de pinabete en Guatemala están reducidos drásticamente, al estar protegida por Cites (Conap, 2006) ha contribuido a que al menos se mantengan en un mismo tamaño desde hace más de 25 años y algunos indicios de recuperación natural se ven. En la parte alta de San Marcos, la mejor señal, de la recuperación de estas áreas es que el mayor factor de tensión sobre estos ecosistemas, que fue el pastoreo de ovejas, ha disminuido drásticamente, el número de cabezas de ganado ovino en el área llegó a alcanzar hasta 2 millones en el siglo XIX (MacLeod, 1980), y para el año 2003 para San Marcos únicamente se reportaron 130,871 cabezas que corresponde al $33 \%$ del total a nivel nacional (Instituto Nacional de Estadística, 2005). Además ahora en los bosques de pinabete se prohíbe el ingreso de ovejas. Ello ha favorecido alrededor de los bosques la sucesión ecológica, que es evidente con la regeneración de arbustos (Martínez, 2013). Con las técnicas de restauración esto se puede acelerar y provocar que la recuperación de estos bosques se más acelerada. 
Sin olvidar que la restauración de ecosistemas es un proceso integral, la vegetación y el suelo ejercen una marcada influencia para la recuperación. Por ello, lo que se propone en este documento es que a partir de promover la restauración de la vegetación se mejoren indirectamente las características del suelo lo que puede traer un efecto cascada en la mejora de otras características y con ello el aumento de los bienes y servicios ecosistémicos de estos bosques (Costanza et al., 1997; Gómez, 2004). Asimismo, las condiciones de diálogo con los principales actores sociales y la gobernanza en el tema de bosques, dan la viabilidad para llevar a cabo un proceso de recuperación (Martínez, Pérez, Rivera, \& Velásquez, 2013). Es importante indicar que acciones como las planteadas aquí ya se están tratando de poner en marcha en el área y tanto las municipalidades, organizaciones gubernamentales y no gubernamentales y las comunidades están jugando un papel principal.

La degradación producida por siglos, que puede comprobarse con en el tamaño actual de los bosques de pinabete, unido al clima del área, hacen que la restauración sea todo un reto, pues junto a lo anterior las especies forestales son de lento crecimiento. Si se toma en cuenta, que cada año la disponibilidad de árboles en almácigo en cantidad y calidad es escasa, pues la capacidad de los viveros locales es baja, el enriquecimiento con especies arbustivas y arbóreas alrededor de bosques de pinabete es lento. Con una proyección de establecimiento anual de al menos 4 ha por año, en 20 años puede haberse recuperado aunque sea un porcentaje pequeño de área. Si la capacidad de los viveros pudiera mejorarse, las metas señaladas podrían superarse.

Esta investigación contribuye a la sistematización de la información de recuperación de bosques en Guatemala, que es importante para estar preparados como país, en las acciones del ámbito mundial, ya que tanto la Convención de Diversidad Biológica como la de Cambio Climático han llegado a la conclusión que la restauración es una de las mejores acciones para la recuperación de bosques. Así se contribuye a alcanzar las metas Aichi de diversidad biológica, en especial la meta 15, planteadas por la Secretaría del Convenio sobre la Diversidad Biológica (2011), a partir de las cuales han resultado compromisos como el Desafio de Bonn (Global Partnership of Forest Landscape Restoration, 2011) que plantea la oportunidad mundial para la restauración de bosques hacia el año 2020 y el compromiso en el foro mundial de bosques de Nueva York en 2014, donde Guatemala se ha comprometido con la restauración de 1.2 millones de ha de 2014 al 2030 (Suding et al., 2015).
Las acciones que se plantean en este documento, para la recuperación de los alrededores de los bosques de pinabete, es solo la parte central de la ejecución de un plan de restauración ecológica, en su totalidad este proceso se integra de varios pasos, Vargas (2007) propone 13, Maginnis y Jackson (2005) 16; en ambos casos los aspectos centrales son las prácticas de campo dentro de las que están el enriquecimiento con especies de la sucesión ecológica del área, tal como se plantea en este trabajo. Por lo que la propuesta de este documento puede adaptarse a cualquiera de los esquemas mencionados. Tomando en consideración que en la teoría se proponen varias formas de recuperación de ecosistemas, algunas asociadas hacia la conservación y otras hacia la oferta de bienes y servicios, siguiendo las ideas de Suding y colaboradores (2015) se proponen cuatro principios que se tienen que alcanzar: (a) la restauración debe incrementar la integridad ecológica, (b) la restauración tiene que ser sustentable en el largo plazo, (c) la restauración es informada por el pasado y futuro de los ecosistemas, y, (d) los beneficios de la restauración involucran a la sociedad.

\section{Agradecimientos}

Se agradece al personal de la Oficina Forestal de la Municipalidad de Ixchiguán, por la colaboración en esta investigación. A los comunitarios de Ixchiguán y Canatzaj, por su colaboración en el campo. A Sergio Osorio encargado de Pinabete Región VI-3 del Inab, por el acompañamiento en los trabajos de campo. Al Ing. Agr. Carlos Godínez por la elaboración de los dibujos. Se agradece al Fondo de Ciencia y Tecnología (Fonacyt) del Consejo Nacional de Ciencia y Tecnología (Concyt) de Guatemala, por el apoyo económico brindado para la obtención de la información a través del proyecto Fodecyt 046-2012.

\section{Referencias}

Andersen, U. S., Prado, J. P., Sorensen, M., Nielsen, U. B., Olsen, C. S., Nielsen, C., ... Kollmann, J. (2008). Conservation through utilization: A case study of the vulnerable Abies guatemalensis in Guatemala. Oryx, 42(2), 206-213. doi:10.1017/ S0030605308007588

Bradshaw, A. D. (2002). Introduction and Philosophy. En M. R. Perrow \& A. J. Davy (Eds.), Handbook of ecology restoration, (Vol.1 Principles of 
Restoration, pp. 3-9).). United Kingdom: Cambridge Press.

Comisión Nacional Forestal. (2013). Linea base de degradación de tierras y desertificación. Informe final. Zapopan, Jalisco, México: Comisión Nacional Forestal y Universidad Autónoma de Chapingo. Recuperado de http://www.semarnat.gob.mx/sites/ default/files/documentos/fomento/documentos/degradacion-tierras-desertificacion2.pdf

Consejo Nacional de Áreas Protegidas. (2006). Convención sobre el Comercio Internacional de Especies Amenazadas de Fauna y Flora Silvestres. Listado de especies de flora y fauna silvestres CITES de Guatemala. Guatemala: Autor, CITES.

Consejo Nacional de Áreas Protegidas. (2008). Diagnóstico del contexto institucional, ambiental y forestal para la conservación y fomento de los bosques naturales y plantaciones de Pinabete (Abies guatemalensis Rehder). Guatemala: Autor.

Consejo Nacional de Áreas Protegidas, Instituto Nacional de Bosques. (1999). Diagnóstico de las poblaciones naturales de pinabete (Abies guatemalensis R.) en Guatemala y estrategia para su conservación. Guatemala: Autor.

Costanza, R., d'Arge, R., de Groot, R., Farber, S., Grasso, M., Hannon, B., ... van den Belt, M. (1997). The value of the world's ecosystem services and natural capital. Nature, 387, 253260.

Fassbender, H. W. (1975). Química de suelos con énfasis en los suelos de América Latina. Turrialba, Costa Rica: Instituto Interamericano para la Agricultura.

Gall, F. (1999). Diccionario Geográfico de Guatemala (Tomo 2) [CD]. Guatemala: Instituto Geográfico Nacional.

Global Partnership of Forest Landscape Restoration (2011). The Bonn Challenge and landscape restoration. Washington, DC: UICN. Recuperado de http://www.forestlandscaperestoration.org/ sites/default/files/topic/the bonn_challenge.pdf

Gómez, L. (2004). Valoración de bienes y servicios ambientales en los bosques naturales de pinabete (Abies guatemalensis Rehder), en tres regímenes de propiedad, de los municipios san José Ojetenam, San Cristóbal Ixchiguán y Concepción
Tutuapa, San Marcos (Tesis de licenciatura). Universidad de San Carlos de Guatemala, Centro Universitario de Noroccidente, Quetzaltenango, Guatemala.

Gordillo, E., Muñoz, M. del C., \& Estrada, J. C. (2005). Libro de texto universitario sobre Historia de Guatemala: Época Colonial, 15241821 (Inf-2005-025). Guatemala: Universidad de San Carlos de Guatemala, Dirección General de Investigación, Instituto de Investigaciones Históricas, Antropológicas y Arqueológicas.

Hobbs, R. J., Higgs, E., Hall, C. M., Bridgewater, P., Chapin III, F. S., Ellis, E. C., ... Yung, L. (2014). Managing the whole landscape: Historical, hybrid, and novel ecosystems. Frontiers in Ecology and the Environment, 12(10), 557-564. doi:10.1890/130300

Ignosh, J. P. \& Kilgore, M. A. (2005). Pinabete one opportunity towards the sustainable development of the Guatemalan highlands. Staff paper series No. 185, Department of Forest Resources. Minesota, USA: The University of Minnesota. Recuperado de ww2.bse.vt.edu/green/.../cfans asset_184716.pdf

Instituto Nacional de Estadística. (2005). IV censo nacional agropecuario. Número de fincas censales, existencia animal, producción pecuaria y características complementarias de la finca censal y del productor(a) agropecuario (Tomo 4). Guatemala: Autor.

Jaramillo-Correa, J. P., Aguirre-Planter, E., Khasa, D. P., Eguiarte, L. E., Piñero, D., Furnier, J. R., \& Bousquet, J. (2008). Ancestry and divergence of subtropical montane forest isolates: Molecular biogeography of the genus Abies (Pinaceae) in southern Mexico and Guatemala. Molecular Ecology, 17(10), 2476-2490. doi: 10.1111/j.1365294X.2008.03762.

Kass, D. C. (1998). Fertilidad de suelos. San José, Costa Rica: Editorial Universidad Nacional a Distancia.

MacLeod, M. J. (1980). Historia socioeconómica de la América Central Española 1520-1720. Guatemala: Piedra Santa.

Maginnis, S., \& Jackson W. (2005). ¿En qué consiste la RPF y cómo se diferencia de los métodos actuales? En Organización Internacional de las 
Maderas Tropicales. Restaurando el paisaje forestal: Introducción al arte y ciencia de la restauración de paisajes forestales (Serie técnica OIMT No. 23, pp. 16-26). Japón: Organización Internacional de las Maderas Tropicales, Unión Mundial para la Naturaleza.

Martínez, J. V. (2013). Sucesión ecológica secundaria alrededor de parches de bosque con pinabete (Abies guatemalensis Rehder) en San Marcos, Guatemala (Tesis de doctorado). Universidad Nacional de Costa Rica, Instituto Tecnológico de Costa Rica, Universidad Estatal a Distancia, San José, Costa Rica.

Martínez, J. V., Pérez, M., Rivera, C. O., \& Velásquez, M. M. (2013). Integración del conocimiento científico y el tradicional en los procesos de restauración de los bosques de pinabete (Abies guatemalensis Rehder), San Marcos, Guatemala. Tikalia, 31(1), 73-91.

Matteucci, S. D. \& Colma, A. (1982). Metodología para el estudio de la vegetación (Serie de biología, Monografía No. 22). Washington, D.C.: Organization of American States.

Meneses-Tovar, C. L. (2011). El índice normalizado diferencial de la vegetación como indicador de la degradación del bosque. Unasylva, 238(622), 39-46.

Montenegro, A. L., \& Vargas R., O. (2008). Caracterización de bordes de bosque altoandino e implicaciones para la restauración ecológica en la Reserva Forestal de Cogua (Colombia). Revista Biología Tropical, 56(3), 1543-1556.
Secretaría del Convenio sobre la Diversidad Biológica. (2011). Plan Estratégico para la Diversidad Biológica 2011-2020 y las Metas de Aichi. Montreal, Quebec: PNUMA. Recuperado de https://www. cbd.int/doc/strategic-plan/2011-2020/Aichi-Targets-ES.pdf

Segura, M., Castillo, A., \& Alvarado, A. (2006). Efecto de la fertilización con fósforo sobre la nodulación de Frankia en plantaciones de Alnus acuminata en andisoles de Costa Rica. Agronomía Costarricense, 30(1), 43-52.

Suding, K, Higgs, E., Palmer, M., Callicott, J. B., Anderson, Ch. B., Baker, M., ... Schwartz, K. Z. (2015). Committing to ecological restoration: Efforts around the globe need legal and policy clarification. Science, 348(6235), 638-640. doi: 10.1126/science.aaa4216

Vargas, O. (Ed.). (2007). Guía metodológica para la restauración ecológica del bosque altoandino. Colombia: Facultad de Ciencias, Universidad Nacional de Colombia. Recuperado de http://www. ciencias.unal.edu.co/unciencias/data-file/user_46/ file/Guia\%20Metodologica.pdf

Vila, J., Varga, D., Llausàs, A., Ribas, A. (2006). Conceptos y métodos fundamentales en ecología del paisaje (landscape ecology). Una interpretación desde la geografía. Documents d'Anàlisi Geogràfica, 48, 151-166. Recuperado de http:// web2.udg.edu/aigua/material/Conceptos $\% 20$ $\mathrm{y} \% 20 \mathrm{~m} \% \mathrm{C} 3 \%$ A 9 todos $\% 20$ fundamentales $\% 20$ en $\% 20$ ecolog $\%$ C3\%ADa $\% 20$ del $\% 20$ paisaje DAG_48_2006.pdf 